point $x$ may be written as

where

$$
\delta H / H=(\delta H / H)_{\mathrm{ad}}+V,
$$

$$
V(x)=\int_{0}^{\infty} \frac{\partial}{\partial \varphi}\left(\frac{\delta H}{H}\right)_{\mathrm{ad}} e^{-i \varphi} d \varphi
$$

is the non-adiabatic correction which must be applied to $(\delta H / H)_{\text {ad }}$, the flux variation computed on the basis of adiabatic oscillations. Here $\varphi\left(x, x^{\prime}\right)$ is essentially a measure of the amount of internal thermal energy contained between shells of radii $x^{\prime}$ and $x$. The stability coefficient (Rosseland I949) has also been expressed in terms of the above solution of the $V$-equation. The above theory is applicable to either radiative or convective transfer, or both.

In order to determine the general conditions required for a pulsating star to exhibit the observed phase lag of roughly a quarter period in the emitted flux and the necessary instability for sustained pulsations, the above theory has been applied to a number of schematic variations of $(\delta H / H)_{\text {ad }}$ with $\varphi$. One of these corresponds to the case studied by Eddington (1927), and gives results in general agreement with his. Radiative red-giant models such as those of Schwarzschild and Li Hen (I949) do not yield the correct phase relationships, showing a phase lead of some $70^{\circ}$ to $90^{\circ}$ in the emitted flux. It appears that the only way in which $\delta H / H$ can have a large phase lag at the surface is for $(\delta H / H)_{\text {ad }}$ to experience an abrupt and rather large drop at some point near the surface. The value of the phase lag in the surface value of $\delta H / H$ so obtained depends on the point at which the drop occurs, the heat-storage capacity of the material exterior to this point, and the details of the drop in $(\delta H / H)_{\text {ad }}$.

Such an abrupt drop in $(\delta H / H)_{\text {ad }}$ will also cause the regions exterior to this point to exert a destabilizing influence on the pulsations. It appears, in fact, that for conditions likely to be expected in giant-star models, maximum instability will be attained for a phase lag in $\delta H / H$ at the surface of near a quarter period.

An abrupt drop in the radiative part, at least, of $(\delta H / H)_{\text {ad }}$ can result from (I) a sudden drop in $\gamma$, or (2) a sudden reversal in the sign of the temperature exponent in the opacity law. As noted by Whitney in a private communication and by Schatzman (1956), both of these effects will occur when fully ionized hydrogen is in the process of deionization, provided hydrogen is the principal constituent of the outer layers of the star.

In addition, it appears to be possible to derive a theoretical period-luminosity relation which agrees reasonably well with observation, if $n_{\text {eff }}$ at the point at which $(\delta H / H)_{\text {ad }}$ drops is greater than about 3 or 4 . The assumptions underlying this derivation are (I) that the point at which $(\delta H / H)_{\text {ad }}$ drops is determined by the degree of ionization of hydrogen at this point; (2) that the degree of ionization of hydrogen at this point has the same value in a sequence of homologous stars; and (3) that the phase lag in the surface value of $\delta H / H$ is a homology invariant.

Recent studies by Schatzman (1956) of the convective-zone models of Mme. DumezilCurien (1954) show that $(\delta H / H)_{\text {ad }}$, when both convective and radiative transfer are considered, does indeed drop rather abruptly near the surface of the star, attaining, in fact, a large negative value there. However, it remains to be seen whether this drop is of such character as to yield agreement with observation.

Dumezil-Curien, Mme. P. I954, Ann. Astroph. 17, I97. Eddington, A. S. I927, M. N. 87, 539.

Rosseland, S. I949, The Pulsation Theory of Variable Stars (Oxford: Clarendon Press).

Schatzman, E. 1956, Ann. Astroph. I9, 5I.

Schwarzschild, M. I938, Z. Astroph. I5, I4.

Schwarzschild, M. and Li Hen I949, M. N. rog, 63I.

Woltjer, J., Jr. I936, B. A. N. 8, I7.

Astronomy Department Cornell University,
Ithaca, N. Y.

\section{Cuffey, James. Color-magnitude relations in Messier 53 and N.G.C. 7492.}

The globular clusters Messier 53 and N.G.C. 7492 are of particular interest in representing extreme differences in physical structure and stellar distribution. If the differences result from age, or from evolutionary differences, the color-magnitude relations might be expected to show significant differences, but this is found not to be the case. The color-magnitude relations, obtained from Mt. Wilson, Palomar, and Link plates, are strikingly similar and resemble that of Messier 3. The typical red giant branch appears in both cases, although with few stars redder than $P-V=$ 0.9. A well-defined horizontal branch extending to $P-V=-0.3$ is present in both clusters. The main sequences lie below the limiting magnitude, I9.5. Variable stars appear at $V=17.05$ in Messier 53, and at 16.95 in N.G.C. 7492. Preliminary distance estimates are: Messier 53, $26 \mathrm{kpc}$, and N.G.C. 7492, $24 \mathrm{kpc}$.

Goethe Link Observatory Indiana University, Bloomington, Ind. 